\title{
Analysis and Numerical Simulation of A Nonlinear Diffusion Equation
}

\author{
H. R. Clark, M. A. Rincon, and D. G. Alfaro Vigo
}

\begin{abstract}
We establish theoretical and numerical analysis such as existence and uniqueness of solutions, asymptotic behavior to the energy and numerical discretization by Finite difference and Finite element methods to a nonlinear diffusion equation with nonlinearities local and nonlocal.
\end{abstract}

Index Terms-Nonlinear diffusion equation, global solution, B-spline polynomial, finite element method, numerical solution.

\section{INTRODUCTION}

Let $\Omega$ be an open, bounded and connect set of $R^{n}$ with smooth boundary $\Gamma$. The objective of this paper is to show the existence, uniqueness and asymptotic stability of the energy of the solutions of equation (1) below, we also present some numerical results related to these topics.

The equations read

$$
\left\{\begin{array}{l}
\theta^{\prime}-M\left(\int_{\Omega} \theta(t) d x\right) \Delta \theta+f(\theta)=0 \\
\theta(x, t)=0 \text { on } \Gamma \times] 0, \infty[ \\
\theta(x, 0)=\theta_{0}(x) \text { in } \Omega
\end{array}\right.
$$

where $M$ and $f$ are real-valued functions and all derivatives of system (1) are considered in the distribution sense.

The nonlocal term in equation (1) is derived by an approach of the Fourier law when, for example, in the case of $\theta=\theta(x, t)$ being the temperature and its measurements are not made locally (pointwise), but on average across the conductor $\Omega$. . Thus, the Fourier law can be given by $\overleftarrow{v}=-\tau(t) \nabla \theta$ where $\tau$ is the function i.e. the thermal diffusivity depends on the total heat in the region $\Omega$. For more detail about that, see [1] and [2].

$$
\tau(t)=M\left(\int_{\Omega} \theta(t) d x\right)
$$

In this work we present some simple contributions to the theoretical analysis of the model investigated in [1] and [3]. Additionally, we develop a numerical method following some ideas introduced in [4], and show some numerical results associated with the asymptotic behavior of the solution of equation (1).

Manuscript received March 9, 2013; revised May 29, 2013.

H. R. Clark is with Instituto de Matemática, Universidade Federal Fluminense, Brazil (e-mail: haroldoclark1@gmail.com).

M. A. Rincon and D. G. Alfaro Vigo are with Instituto de Matemática, Universidade Federal do Rio de Janeiro, Brazil (e-mail: rincon@dcc.ufrj.br, dgalfaro@dcc.ufrj.br).
We shall follow the usual notation for functional spaces, see for instance [5] or [6].

\section{EXISTENCE, UNIQUENESS AND EXPONENTIAL DECAY}

In order to state the main results, we consider some hypotheses on the real-valued functions $M$ and $f$. Namely,

$$
\begin{aligned}
& M \text { is continuos on IR } \\
& \text { and } M(s) \geq M_{0}>0, \forall s \in I R \text {. } \\
& f(0)=0 \text { and } f \text { is Lipschitz continuous } \\
& \text { on IR with a Lipschitz constant L. }
\end{aligned}
$$

Definition 2.1 A global strong solution for the nonlinear initial-boundary value problem (1) is a real-valued function $\theta(x, t)$ defined on $\Omega \times[0, \infty)$ such that

$$
\begin{aligned}
& \theta \in C\left([0, \infty) ; H_{0}^{1}(\Omega)\right) \cap L_{\mathrm{loc}}^{2}\left(0, \infty ; H^{2}(\Omega)\right), \\
& \theta^{\prime} \in L_{\mathrm{loc}}^{2}\left(0, \infty ; L^{2}(\Omega)\right) .
\end{aligned}
$$

The function $\theta$ satisfies the identity integral for all $\varphi \in L^{2}\left(0, T ; H_{0}^{1}(\Omega)\right)$. Moreover, $\theta$ satisfy the initial conditions $(1)_{3}$.

$$
\begin{aligned}
\int_{0}^{\infty}\left[\int_{\Omega} \theta^{\prime} \varphi d x\right. & +M\left(\int_{\Omega} \theta\right) \int_{\Omega} \nabla \theta \cdot \nabla \varphi d x \\
& \left.+\int_{\Omega} f(\theta) \varphi d x\right] d t=0
\end{aligned}
$$

Theorem 2.1 Suppose $\theta_{0} \in H_{0}^{1}(\Omega)$. Then there exists a unique global solution of (1) in the sense of Definition 2.1, provided the hypotheses in (2) hold, and the energy $E(t)=|\theta(t)|^{2}$ satisfies the estimate

$$
E(t) \leq E(0) \mathrm{e}^{\zeta t} \text { for all } t \geq 0
$$

with the constant $\zeta:=2\left(L-M_{0} \lambda_{1}\right)$ where $L$ and $M_{0}$ are defined in (2) and $\lambda_{1}$ represents the first eigenvalue of the Laplace operator.

As a simple consequence of this theorem, we preent a simple condition for the decay of the solution energy in the following corollary.

Corollary 2.1: When $M_{0}>L / \lambda_{1}$, the energy of the solution asymptotically decays to zero as time increases.

Proof of the existence of solutions. We use the Faedo-Galerkin method. Let $\left(w_{i}\right)_{i \in I N}$ be a Hilbertian basis 
of eigenvectors for the Laplace operator in $H_{0}^{1}(\Omega)$. For each $m \in I N$ we obtain the approximate solution

$$
\theta_{m}(x, t)=\sum_{i=1}^{m} g_{i}(t) w_{i}(x)
$$

corresponding to the approximate problem

$$
\begin{aligned}
\left(\theta_{\mathrm{m}^{\prime}}, w\right)- & M\left(\int_{\Omega} \theta_{\mathrm{m}}\right)\left(\Delta \theta_{\mathrm{m}}, w\right) \\
& +\left(f\left(\theta_{\mathrm{m}}\right), w\right)=0, \\
\theta_{\mathrm{m}}(x, 0)= & \theta_{0 \mathrm{~m}}(x) \rightarrow \theta_{0}(x) \text { in } \mathrm{H}_{0}^{1}(\Omega),
\end{aligned}
$$

for all $w \in W_{m}=\operatorname{span}\left\{w_{1}, \ldots, w_{m}\right\}$. Problem (6) has a local solution $\theta_{m}$ in the interval $\left[0, t_{m}\right)$ and its extension to the half-line $[0, \infty)$ is a consequence of the first estimate established below.

Estimate $I$ - Setting $w=2 \theta_{m}$ in (6) we get that

$$
\frac{d}{d t}\left|\theta_{m}\right|^{2}+2 M\left(\int_{\Omega} \theta_{m}\right)\left|\nabla \theta_{m}\right|^{2}+2\left(f\left(\theta_{m}\right), \theta_{m}\right)=0 .
$$

Using the hypothesis (2) we get that

$$
\frac{d}{d t}\left|\theta_{m}\right|^{2}+2 M_{0}\left|\nabla \theta_{m}\right|^{2} \leq 2 L\left|\theta_{m}\right|^{2}
$$

Applying the Poincaré inequality $\lambda_{1}\left|\theta_{m}\right|^{2} \leq\left|\nabla \theta_{m}\right|^{2}$ the Gronwall lemma and the fact that $\theta_{m}\left(0\right.$ converges in $L^{2}(\Omega)$ we arrive at

$$
\left|\theta_{m}(t)\right|^{2} \leq\left|\theta_{m}(0)\right|^{2} \mathrm{e}^{\zeta t} \leq C_{0} \mathrm{e}^{\zeta t}, \quad t \geq 0
$$

with a constant $C_{0}=\sup _{m}\left|\theta_{m}(0)\right|^{2}<\infty$.

Estimate II - Taking $w=-2 \Delta \theta_{m}$ in $(7)_{1}$ it yields

$$
\frac{d}{d t}\left|\nabla \theta_{m}\right|^{2}+2 M\left|\Delta \theta_{m}\right|^{2}=2\left(f\left(\theta_{m}\right), \Delta \theta_{m}\right) \text {. }
$$

Using hypothesis (2) together with the Cauchy-Schwarz, Poincaré and Young's inequalities, we have that for any $\varepsilon>0$

$$
\frac{d}{d t}\left|\nabla \theta_{m}\right|^{2}+\left(2 M_{0}-\varepsilon\right)\left|\Delta \theta_{m}\right|^{2} \leq \frac{L^{2}}{\lambda_{1} \varepsilon}\left|\nabla \theta_{m}\right|^{2} .
$$

Finally, considering $\varepsilon=M_{0}$, integrating in time and using the Gronwall lemma we get

$$
\begin{array}{ll}
\left|\nabla \theta_{\mathrm{m}}(\mathrm{t})\right|^{2} & \leq \mathrm{C}_{1} \mathrm{e}^{\zeta_{1} \mathrm{t}} \\
\int_{0}^{\mathrm{t}}\left|\Delta \theta_{\mathrm{m}}(\mathrm{s})\right|^{2} \mathrm{ds} & \leq \mathrm{C}_{2} \mathrm{e}^{\zeta_{1} \mathrm{t}}, \quad \mathrm{t} \geq 0
\end{array}
$$

where $\zeta_{1}=\frac{L^{2}}{\lambda_{1} M_{0}} \quad, \quad C_{1}=\sup _{m}\left|\nabla \theta_{m}(0)\right|^{2}<\infty \quad$ and $C_{2}=\frac{C_{1}}{M_{0}}$
Estimate III - Setting $w=\theta_{m}^{\prime}$ in $(7)_{1}$, we get

$$
\left|\theta_{\mathrm{m}}^{\prime}\right|^{2}=\mathrm{M}\left(\int_{\Omega} \theta_{\mathrm{m}}\right)\left(\Delta \theta_{\mathrm{m}}, \theta_{\mathrm{m}}^{\prime}\right)-\left(\mathrm{f}\left(\theta_{\mathrm{m}}\right), \theta_{\mathrm{m}^{\prime}}\right)
$$

Using hypothesis (2) together with the Cauchy-Schwarz inequality we obtain

$$
\left|\theta_{m}^{\prime}\right| \leq M\left(\int_{\Omega} \theta_{m}\right)\left|\Delta \theta_{m}\right|+L\left|\theta_{m}\right|
$$

Using estimates (9) and (10) we arrive at

$$
\left\{\int_{0}^{\mathrm{t}}\left|\theta_{\mathrm{m}}^{\prime}(\mathrm{s})\right|^{2} \mathrm{ds}\right\}^{2} \leq \tilde{\mathrm{M}}_{\mathrm{t}} \sqrt{\mathrm{C}_{2}} \mathrm{e}^{\frac{\zeta_{1}}{2} \mathrm{t}}+\mathrm{L} \sqrt{\mathrm{C}_{0}} \sqrt{\frac{\mathrm{e}^{\zeta \mathrm{t}}-1}{\zeta}}
$$

where $\tilde{M}_{\mathrm{t}}=\max _{\mid l \leq \mathrm{K}_{\mathrm{t}}} \mathrm{M}(\mathrm{l})$, with $\mathrm{K}_{\mathrm{t}}=\max \left\{\mathrm{C}_{0}, \mathrm{C}_{0} \mathrm{e}^{\zeta \mathrm{t}}\right\}$

The estimates (9), (10) and (12) allows us to pass to the limit in the approximate solutions $\theta_{m}$. In fact, for any $T>0$ we can extract a subsequence $\left(\theta_{\mu}\right)_{\mu \in \mathrm{N}}$ of the sequence of approximation $\left(\theta_{m}\right)_{m \in N}$, such that

$$
\begin{array}{ll}
\theta_{\mu} & \rightarrow \theta \quad \text { weak in } L^{2}\left(0, T ; H_{0}^{1}(\Omega) \cap H^{2}(\Omega)\right) \\
\theta_{\mu}^{\prime} & \rightarrow \theta^{\prime} \quad \text { weak in } L^{2}\left(0, T ; L^{2}(\Omega)\right) .
\end{array}
$$

Morever, since the injection of $H_{0}^{1}(\Omega) \cap H^{2}(\Omega)$ into $H_{0}^{1}(\Omega)$ is continuous we also have that $\theta \in C\left([0, T] ; H_{0}^{1}(\Omega)\right)$ and $\theta(0)=\theta_{0}$.

The convergence (13) are sufficient to pass to the limit as $\mu \rightarrow \infty$ in the first two terms of the approximate system (7), but is not sufficient to analyse the nonlinear terms. Moreover, we need convergence in $L^{2}\left(0, T ; L^{2}(\Omega)\right)$ in order to determine the limit of these terms

$$
\mathrm{M}\left(\int_{\Omega} \theta_{\mathrm{m}}(\mathrm{t}) \mathrm{dx}\right) \Delta \theta_{\mathrm{m}}(\mathrm{x}, \mathrm{t}) \text { and } \mathrm{f}\left(\theta_{\mathrm{m}}(\mathrm{x}, \mathrm{t})\right)
$$

as the injection of $H_{0}^{1}(\Omega) \cap H^{2}(\Omega)$ into $H_{0}^{1}(\Omega)$ is continuous and compact then (13) enable us to apply compactness result, cf. Aubin [6] or Lions [7], and thus we can extract a subsequence of $\left(\theta_{\mu}\right)_{\mu \in \mathrm{N}}$, which still shall be reprepresented by $\left(\theta_{\mu}\right)_{\mu \in \mathrm{N}}$, such that

$$
\theta_{\mu} \rightarrow \theta \quad \text { strongly } L^{2}\left(0, T ; H_{0}^{1}(\Omega)\right)
$$

and whence we get

$$
\theta_{\mu} \rightarrow \theta \quad \text { a.e. in } \quad \Omega \times[0, \mathrm{~T}] .
$$

Analysis of the term $M\left(\int_{\Omega} \theta_{\mu} d x\right) \Delta \theta_{\mu}$

We will proof the following convergence:

$$
\begin{aligned}
& \int_{0}^{T}\left(M\left(\int_{\Omega} \theta_{\mu}(t) d x\right) \nabla \theta_{\mu}(t), \nabla v(t)\right) d t \\
& \rightarrow \int_{0}^{T}\left(M\left(\int_{\Omega} \theta(t) d x\right) \nabla \theta(t), \nabla v(t)\right) d t
\end{aligned}
$$


as $\mu \rightarrow \infty$ for all $v \in L^{2}\left(0, T ; H_{0}^{1}(\Omega)\right)$. Note that

$$
\begin{aligned}
\int_{0}^{T}\left(M\left(\int_{\Omega} \theta_{\mu}\right) \nabla \theta_{\mu}-M\left(\int_{\Omega} \theta\right) \nabla \theta, \nabla v\right) \\
=\int_{0}^{T}\left[M\left(\int_{\Omega} \theta_{\mu}-\int_{\Omega} \theta\right)\right]\left(\nabla \theta_{\mu}, \nabla v\right) \\
+\int_{0}^{T}\left(M\left(\int_{\Omega} \theta\right)\left[\nabla \theta_{\mu}-\nabla \theta\right], \nabla v\right) . \\
\text { As } \theta_{\mu}(t) \in L^{2}(\Omega)^{\circ} L^{1}(\Omega) \text { then } \\
\left|\int_{\Omega} \theta_{\mu}(t) d x\right|_{\mathbb{R}} \leq C\left|\theta_{\mu}(t)\right| \leq C
\end{aligned}
$$

From this, (15), Lebesgue dominated convergence theorem and continuity of $M$, it results

$$
\left|M\left(\int_{\Omega} \theta_{\mu}(t) d x\right)-M\left(\int_{\Omega} \theta(t) d x\right)\right|_{I R} \rightarrow 0
$$

as $\mu \rightarrow \infty$. By Cauchy-Schwartz inequality and (10), we have

$$
\left|\left(\nabla \theta_{\mu}, \nabla \mathrm{v}\right)\right|_{\mathbb{R}} \leq \frac{1}{2}\left|\nabla \theta_{\mu}\right|^{2}+\frac{1}{2}|\nabla v|^{2} \leq C
$$

From this and precedent convergence, it yields

$$
\int_{0}^{T}\left[\mathrm{M}\left(\int_{\Omega} \theta_{\mu}\right)-\mathrm{M}\left(\int_{\Omega} \theta\right)\right]\left(\nabla \theta_{\mu}, \nabla \mathrm{v}\right) \rightarrow 0
$$

as $\mu \rightarrow \infty$. Now we analyze the last term on the right-hand side of (17). Initially, note that

$$
\left|M\left(\int_{\Omega} \theta(t) d x\right)\right|_{I R} \leq C \text { for all } t \geq 0
$$

and by Cauchy-Schwarz inequality, we have

$$
\begin{aligned}
\left|\left(\left[\nabla \theta_{\mu}-\nabla \theta\right], \nabla v\right)\right|_{I R} & \leq\left|\nabla \theta_{\mu}-\nabla \theta\right||\nabla v| \\
& \leq C \mid \nabla \theta_{\mu}-\nabla \theta .
\end{aligned}
$$

From (14) we have $\theta_{\mu} \rightarrow \theta$ in $L^{2}\left(0, T ; H_{0}^{1}(\Omega)\right)$ as $\mu \rightarrow \infty$. Thus

$$
\int_{0}^{T}\left(M\left(\int_{\Omega} \theta d x\right)\left[\nabla \theta_{\mu}-\nabla \theta\right], \nabla v\right) d t \rightarrow 0
$$

as $\mu \rightarrow \infty$. Inserting (19) and (18) into (17) we conclude that (16) is fact.

Analysis of the term $f\left(\theta_{\mu}\right)$ : As $\left(\theta_{\mu}\right)$ is bounded in $L^{2}\left(0, T ; L^{2}(\Omega)\right) \quad$ and $\quad f \in C^{0}(I R) \quad$ then $\left(f\left(\theta_{\mu}\right)\right)$ is bounded in $L^{2}\left(0, T ; L^{2}(\Omega)\right)$.

From this and (15), it yields

$$
\mathrm{f}\left(\theta_{\mu}\right) \rightarrow \mathrm{f}(\theta) \text { a.e. in } \Omega \times[0, \mathrm{~T}] .
$$

From (13), (20) and Lions' lemma (cf. [6], ch. I, Lemma 1.3), we get

$$
f\left(\theta_{\mu}\right) \rightarrow f(\theta) \text { weak in } \mathrm{L}^{2}\left(0, \mathrm{~T} ; \mathrm{L}^{2}(\Omega)\right)
$$

Taking to the limit $\mu \rightarrow \infty$ in (6), and using the convergence (16) and (21) we obtain at least one function $\theta$ satisfying the identity integral in (4). The regularities in (3) are given by estimates (12) and (10)

Uniqueness of solutions. The uniqueness of the global solution can be obtained using the energy method.

Asymptotic behavior. The aim now is to prove the inequality (4). Taking the scalar product of $L^{2}(\Omega)$ on both sides of (1) ${ }_{1}$ with $\theta$ and using the hypotheses (2), we get

$$
E^{\prime}(t)+M_{0}|\nabla \theta(t)|^{2} \leq L|\theta(t)|^{2},
$$

where $L>0$ is given by $|f(\xi)| \leq L|\xi|$ for all $\xi \in I R$ and $\quad E(t)=\frac{1}{2}|\theta(t)|^{2}$. As $\theta(t) \in H_{0}^{1}(\Omega)$ then $\lambda_{1}|\theta(t)|^{2} \leq|\nabla \theta(t)|^{2}$, where $\lambda_{1}$ is the first eigenvalue of the Laplace operator. Thus, from (22) we obtain

$$
E^{\prime}(t)+2\left(M_{0} \lambda_{1}-L\right) E(t) \leq 0 \text { for all } t \geq 0
$$

From this we have that (5) follows.

\section{Approximation SOLUtion}

To obtain the numerical approximate solutions we use both the finite element method and the finite difference method. Moreover, some numerical experiments are presented for analysis of the model.

Substituting (6) into the approximate system (7) and taking $w=w_{j}(x) \in V_{m}$, we obtain,

$$
\begin{aligned}
& \sum_{i=1}^{m} g_{i}^{\prime}(t)\left(w_{i}, w_{j}\right)+M\left(\sum_{k=1}^{m} g_{k}(t) \int_{0}^{L} w_{k}(x) d x\right) \\
& \sum_{i=1}^{m} g_{i}(t)\left(\frac{\partial w_{i}}{\partial x}, \frac{\partial w_{j}}{\partial x}\right)+\left(f\left(\sum_{i=1}^{m} g_{i}(t) w_{i}\right), w_{j}\right)=0,
\end{aligned}
$$

We define the square matrix $A$ and $B$ of order $m \times m$, the vector $C\left(f\left(\theta_{m}(t)\right)\right.$ of order $m \times 1$, the real number $M_{k}$ and the real function $\hat{g}(t)$ by;

$$
\begin{aligned}
& A_{i j}=\int_{0}^{L} w_{i} w_{j} ; B_{i j}=\int_{0}^{L} \frac{\partial w_{i}}{\partial x} \frac{\partial w_{j}}{\partial x} \\
& \hat{g}(t)=\sum_{k=1}^{m} \alpha_{k} g_{k}, \quad \alpha_{k}=\int_{0}^{L} w_{k}(x) \\
& C_{j}\left(f\left(\theta_{m}\right)\right)=\int_{0}^{L} f\left(\sum_{i=1}^{m} g_{i} w_{i}\right) w_{j} ;
\end{aligned}
$$

Substituting (23) in (22), we obtain the system 


$$
\begin{aligned}
& \sum_{i=1}^{m} g_{i}^{\prime}(t) A_{i j}+M(\hat{g}(t)) \sum_{i=1}^{m} g_{i}(t) B_{i j} \\
& \quad+C_{j}\left(f\left(\theta_{m}\right)=0, \text { for } \quad j=1, \cdots, m\right.
\end{aligned}
$$

Since that, $A$ and $B$ are symmetric matrices, we get the following nonlinear ordinary differential system;

$$
A g^{\prime}(t)+M(\hat{g}(t)) B g(t)+C\left(f\left(\theta_{m}\right)\right)=0
$$

where $g(0)=g_{0}$ is the initial data and $g(t)=\left[g_{1}(t), \ldots,\left(g_{m}(t)\right]^{t}\right.$ is the vector to be determined at each time $t$.

\section{Finite Element Method}

To calculate the matrices of the nonlinear system (24), we need to introduce the basis function $w_{i} \in V_{m}$. Note in (??) trizes) that the vector $M$ and the function $C\left(f\left(\theta_{m}\right)\right)$ are dependent on the variable $t$, then to get a better precision of the approximate solutions we will use the B-splines as basis function to define the subspace $V_{m}$, which are cubic splines. See, for instance in [4], the definition and a procedure to change the B-spline to satisfy the Dirichlet boundary condition.

Thanks to definition the base function, we can to calculate the values of matrices $A$ and $B$.

Approximation of the nonlinear term $C\left(f\left(\theta_{m}(t)\right)\right)$ :

The nonlinear term is approximated linearly in each interval $\left[x_{j}, x_{j+1}\right]$ as follows:

$$
\begin{aligned}
& C_{j}(t)=C_{j}\left(f\left(\theta_{m}(t)\right)=\int_{x_{j}}^{x_{j+1}} f\left(\theta_{m}(x)\right) w_{j}\right. \\
& =\frac{1}{2} \int_{x_{j}}^{x_{j+1}}\left(f\left(\theta_{m}\left(x_{j}\right)\right)+f\left(\theta_{m}\left(x_{j+1}\right)\right)\right) w_{j} \\
& =\frac{1}{2}\left(f\left(\theta_{m}\left(x_{j}\right)\right)+f\left(\theta_{m}\left(x_{j+1}\right)\right)\right) \int_{x_{j}}^{x_{j+1}} w_{j}
\end{aligned}
$$

Using the definition of the base function $w_{j}$, we can calculate the vector components $C_{j}(t)$.

Calculation of the term $M(\hat{g}(t))$ : Analogously, using the definition of the base function $w_{j}$ and the definition of real number $\alpha_{k}$ in (24), then we can calculate de function $\hat{g}(t)$ as follows;

$$
\begin{aligned}
\hat{g}(t) & =h\left\{g_{1}(t)+\frac{11}{4} g_{2}(t)+\frac{3}{2} \sum_{k=3}^{m-2} g_{k}(t)\right. \\
& \left.+\frac{11}{4} g_{m-1}(t)+g_{m}(t)\right\} .
\end{aligned}
$$

\section{FinITE DifFERENCE METHOD}

For the nonlinear ordinary differential system (25) with the matrices characteristics (dependent on the variables $x$ and $t$ ) to obtain the solution is not always possible in continuous time. So, we will apply a numerical method to determine the approximated solution for this system, using the approximate implicit Crank-Nicolson method (see, for instance, [9]).

From the temporal discretization and considering $\theta^{n}=\theta\left(t_{n}\right)$ we define the the approximation and the first derivative of the time, by

$$
g^{n+\frac{1}{2}}=\left(g^{n+1}+g^{n}\right) / 2, \quad g^{\prime}(t)_{t_{n}}=\left(g^{n+1}-g^{n}\right) / \Delta t
$$

For the system (25) at the discrete mesh points $t_{n}=n \Delta t$, using approximation above, we obtain the following the discrete system:

$$
A\left(\frac{g^{n+1}-g^{n}}{\Delta t}\right)+M^{n+\frac{1}{2}} B g^{n+\frac{1}{2}}+C^{n+\frac{1}{2}}=0
$$

where, we are denoting $M^{n}=M\left(g^{n}\right)$ and $C^{n}=C_{j}\left(f^{n}\right)=C\left(f\left(\theta_{m}^{n}\right), w_{j}\right)$.

Multiplying the above equation by $2 \Delta t$ then, we can rewrite the equation above as;

$$
\left\{\begin{array}{l}
\left(2 A+\Delta t M^{n+1} B\right) g^{n+1}= \\
\left(2 A-\Delta t M^{n} B\right) g^{n}-\Delta t\left(C^{n+1}+C^{n}\right) \\
g(0)=g^{0} \text { for } n=0,1, \cdots N
\end{array}\right.
$$

The nonlinear iterative system (27) can be implemented, for example, using the Newton's Method. The solvability of this system yields the vector solution $g^{1}=\left(g_{1}^{1}, g_{2}^{1}, \cdots, g_{m}^{1}\right)$ at time $t=t_{1}=\Delta t$. Therefore, for $n=1,2, \cdots, N$ we will get by the same procedure the values of $g^{n}=\left(g_{1}^{n}, g_{2}^{n}, \cdots, g_{m}^{n}\right)$.

\section{NUMERICAL SiMULATIONS}

In this section, attention is turned to the computation of approximate solutions.

The following example concerns the accuracy and convergence rate of the numerical method. With this aim in mind, we compare the exact and approximate solutions of an equation obtained from (1) by adding a non zero right hand side unction $g(x, t)$. We test two different situations: (a) the linear case where the function $M(s) \equiv 1$ and $f(\theta) \equiv 0$, and (b) a non linear case with $M(s)=0.5+s^{2}$ and the nonlinearity $f(\theta)=\theta^{3}$.

In both cases, we consider an spatial interval $[0, L]=[0,1]$, a final time $T=1$, the initial condition $\theta_{0}(x)=\sin (\pi x)$ and choose the function $g(x, t)$ in such a way that the exact solution is given by $\theta(x, t)=\mathrm{e}^{t} \sin (\pi x)$.

The numerical solutions are computed for different discretizations and the corresponding numerical errors are calculated in the $\mathrm{L}^{\infty}\left(0, \mathrm{~T} ; \mathrm{L}^{2}[0,1]\right)$ norm. It is remarkable that the results for both cases are qualitatively similar. For case (b), they are summarized in Table I. 
TABLE I: NUMERICAL ERRORS AND CONVERGENCE RATE FOR THE NON LINEAR CASE (B).

\begin{tabular}{|l|l|l|c|}
\hline$\Delta t$ & $\Delta x$ & Error & Convergence rate \\
\hline $1 \mathrm{e}-4$ & 0.0015625 & $9.4447 \mathrm{e}-07$ & - \\
\hline & 0.0007812 & $2.3612 \mathrm{e}-07$ & 2.0000 \\
\hline & 0.0003906 & $5.9037 \mathrm{e}-08$ & 1.9999 \\
\hline & 0.0001953 & $1.4774 \mathrm{e}-08$ & 1.9985 \\
\hline $1 \mathrm{e}-5$ & 0.0015625 & $9.4446 \mathrm{e}-07$ & - \\
\hline & 0.0007812 & $2.3612 \mathrm{e}-07$ & 2.0000 \\
\hline & 0.0003906 & $5.9029 \mathrm{e}-08$ & 2.0000 \\
\hline & 0.0001953 & $1.4753 \mathrm{e}-08$ & 2.0004 \\
\hline
\end{tabular}

It is worth noticing that the convergence rate of the proposed numerical method for the linear case is known to be quadratic. Moreover, our numerical results suggest that a similar convergence rate is also valid in the non linear case. This result illustrates that the proposed numerical method can be readily used for the computation of accurate approximate solutions to equation (1).

The next example is related to the asymptotic behavior, as $t$ goes to infinity, of the solutions of equation (1) with the nonlinearity $f(\theta)=a\left(\theta^{3}-\theta\right)$. We consider two cases, in the local case (a) the function $M(s) \equiv 1$, and in the nonlocal case (b) $M(s)=\mathrm{e}^{s}$.

In case (a) equation (1) is known as the Chafee-Infante equation. The asymptotic behavior of the solutions in this case is well understood and was thoroughly studied in [10]. They established that when the parameter $a$ passes through an eigenvalue of the laplacian a bifurcation occurs.

In Fig. 1 we present the bifurcation diagram representing the dependence of the asymptotic solution mass when the parameter $a$ changes in the interval $[5,15]$. Our results suggest that in the nonlocal case (b) a bifurcation occurs at the same value $a_{\text {crit }}=\lambda_{1}=\pi^{2}$ as for the Chafee-Infante equation.

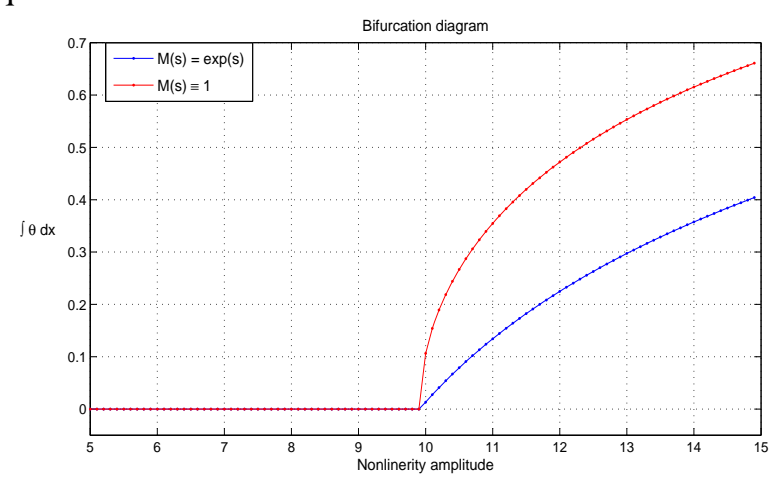

Fig. 1. First bifurcation for the Chaffee-Infante equation in the local and nonlocal cases.

\section{REFERENCES}

[1] M. Chipot and B. Lovat, "On the Asymptotic Behaviour of Some Nonlocal Problems,” Positivity 3, pp. 65-81, 1999.

[2] M. A. Rincon, J. Limaco, and I. S. Liu, "A Nonlinear Heat Equation with Temperature-Dependent Parameters," Mathematical Physics Electronic Journal, vol. 12, $N^{o} 5$, pp. 1-21, 2006.

[3] S. B. de Menezes, "Remarks on existence, uniqueness and periodic solution for a nonlocal parabolic problem," I. J. of Mathematics and Mathematical Sciences, Article ID 82654, pp. 1-10, 2006.

[4] M. G. Teixeira, I. S. Liu, and M. A. Rincon "Numerical Analysis of Quenching - Heat Conduction in Metallic Materials,” Applied Mathematical Modelling, vol. 33, Fasc. 5, pp. 2464-2473, 2009.

[5] H. Brezis, Analise Fonctionnelle. Théorie et applications, Dunod, Paris, 1999.

[6] J. L. Lions, Quelques méthodes de résolution des problèmes aux limites non-linéaires, Dunod, Paris, 1960.

[7] J. P. Aubin, "Un théorème de compacité," C. R. A. Sci. Paris, t. 256, pp. 5042-5044, 1963.

[8] H. R. Clark, M. A. Rincon, and A. Silva, "Analysis and numerical simulation of viscous Burgers equation," Numerical Functional Analysis and Optimization, vol. 32, no. 7, pp. 1-22, 2011.

[9] H. R. Clark, M. A. Rincon, and R. D. Rodrigues, "Beam Equation with Weak-Internal Damping in Domain with Moving Boundary," Applied Numerical Mathematics, vol. 47, $N^{o}$ no. 2, pp. 139-157, 2003.

[10] N. Chafee and E. Infante, "A bifurcation problem for a nonlinear parabolic equation," J. Appl. Anal., no. 4, 1974, pp. 17-37.

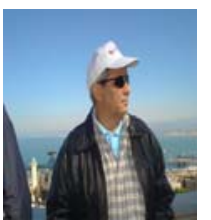

Haroldo Rodrigues Clark was graduated from Federal University of Piauí, Brazil (1981) and obtained a Ph.D. in Mathematics from the Federal University of Rio de Janeiro, Brazil. He is currently assistant professor at the Federal University Fluminense, Rio de Janeiro in Brazil. His research interest is mainly in applied mathematics with focus on analysis of partial differential equations.

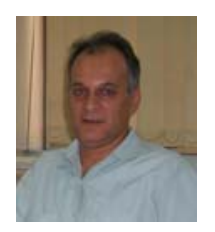

Mauro A. Rincon was graduated from São Paulo State University, Brazil (1983) and obtained a Ph.D. in Mathematics from the Federal University of Rio de Janeiro, Brazil. He is currently assistant professor at the Federal University of Rio de Janeiro in Brazil. His research interest is mainly in applied mathematics with focus on numerical analysis and numerical solution/analysis of partial differential equations.

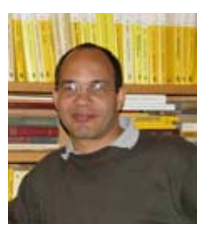

Daniel G. Alfaro Vigo was graduated from Moscow State University (1991) and obtained a Ph.D. in Mathematics from the National Association of Pure and Applied Mathematics (IMPA, 2004). He is currently assistant professor at the Federal University of Rio de Janeiro in Brazil. His research interest is mainly in applied mathematics with focus on wave propagation in heterogeneous media and numerical solution/analysis of partial differential equations. 